www.nature.com/pj

\title{
The systematic study of the microstructure of crosslinked copolymers from siloxane macromonomers and methacrylates by changes in composition and components
}

\author{
Polymer Journal (2012) 44, 301-305; doi:10.1038/pj.2011.143; published online 25 January 2012
}

\section{INTRODUCTION}

Polymerization-induced phase separation ${ }^{1}$ is a very convenient method to create microheterophase structures from a homogeneous solution containing reactive monomeric compounds and polymeric compounds. The technique has been reported in many fields, including in the polymerizations of monomer/polymer mixtures ${ }^{2}$ and hydrogels. ${ }^{3}$

While progress has been made in this field, many radically polymerizable silicone compounds have been developed for use in silicone polymer products, especially with respect to medical devices, such as contact lenses, intraocular lenses and artificial lungs. In the course of improving the limitations associated with silicone, such as fragility and hydrophobicity, the introduction of silicone compounds with long Si-O-Si chains (for example, polydimethylsiloxane (PDMS)) to the polymer backbone have been developed, which leads to microheterophase structure of polymer. One of these approaches is the introduction of PDMS chains by condensation reactions with rigid segments, such as polyamide and polyimide groups, to create multi-block copolymers. ${ }^{4,5}$ Another approach is the polymerizationinduced phase separation method that consists of radical copolymerization of methacrylates with macromers that contain radically polymerizable groups at the end of the PDMS chain and crosslinkers that have multiple radically polymerizable groups.

With the latter approach, an amphiphilic co-network created from the combination of PDMS macromonomer and hydrophilic vinyl monomer ${ }^{6,7}$ has been reported, as has been the crosslinked copolymer from PDMS macromers and hydrophobic vinyl mono- mers. ${ }^{8,9}$ Previous research on the amphiphilic network focused on analysis of the structure or dynamics for a specific polymer composition. Research on hydrophobic crosslinked copolymers focused on the properties of various polymerizable groups at the PDMS tail end. Furthermore, as far as we know, no articles have been published that investigated the microstructure of PDMS-bearing copolymers at the molecular level with solid-state NMR.

In the radical copolymerization approach, a systematic study is desired for the relationship between composition and physical properties as a function of the various monomers and PDMS macromonomers of various molecular weight $\left(M_{\mathrm{n}}\right)$. However, no articles using such an approach were found. We have been studying crosslinked copolymers produced from siloxane macromonomers with varied PDMS chain lengths, various methacrylate monomers and crosslinkers. As part of this study, we have already reported the high performance of products created using the polymerization-induced phase separation method that exhibit microheterophase structure. The materials showed very high oxygen permeability and durability when compared with a material with a low- $M_{\mathrm{n}}$ silicone constituent and that was as uniform a structure as contact lens material. ${ }^{10}$ In addition, it has been reported that the copolymerization of the perfluoroalkyl ester group-bearing monomer with PDMS-containing material caused an increase in oxygen permeability. ${ }^{11}$ However, further analysis of microstructures of the copolymers is required at the molecular level to develop a copolymer material with superior characteristics.

In this study, we used ${ }^{13} \mathrm{C}$ solid-state $\mathrm{CP}$ MAS NMR measurements of the aforemen- tioned copolymers because it provides finegrain information with respect to the molecular mobility or relaxation time, which enables analysis of the internal structure of heterogeneous polymer composites. ${ }^{12-14}$ We chose methylmethacrylate (MMA) and trifluoroethylmethacrylate (TFEMA) as the methacrylates because they have been used as optical materials in medical materials, such as contact lenses, due to their high glass transition temperature $\left(T_{\mathrm{g}}\right)$ and transparency. TFEMA also has a low refractive index, similar to that of PDMS, and oxygen permeability was also considered. We believe that our approach to investigate the relationship between copolymer compositions and their physical properties with solid-state NMR will contribute greatly to the new field of the study of polymerization-induced phase separation products.

\section{EXPERIMENTAL PROCEDURE}

Materials and detailed procedures for the preparation of the crosslinked copolymers from methacrylates/PDMS macromonomers and NMR measurements are provided in the Supplementary Information section. The molecular weights of the PDMS macromonomers are listed in Supplementary Table S1 in the Supplementary Information. Chemical structures of the monomers and crosslinker are shown in Figure 1. The compositions of the samples are shown in Table 1.

\section{RESULTS AND DISCUSSION \\ CP-MAS spectra}

Examples of the obtained CP-MAS spectra and assignments of the signals are shown in Figure 2. The peak patterns differ depending on the chemical structure of the monomers. In addition, the intensity at 1.5 p.p.m. (a) of specimen M147, with a PDMS $M_{\mathrm{n}}$ of 1700 , 
<smiles>C=C(C)C(=O)OC</smiles>

MMA<smiles>C=C(C)C(=O)OCCC[Si](C)(C)O[Si](C)(C)O[Si](C)(C)C(C)CCOC(=O)C(=C)C</smiles>

Figure 1 Chemical structures of the monomers and crosslinker.

Table 1 Composition a of the specimens employed in this study

\begin{tabular}{|c|c|c|c|c|c|c|c|}
\hline \multirow[b]{2}{*}{ Specimen } & \multicolumn{2}{|c|}{ Acrylic monomer } & \multicolumn{2}{|c|}{ PDMS Macromonomer } & \multicolumn{2}{|c|}{$P D M S^{b}$} & \multirow[b]{2}{*}{ Appearence } \\
\hline & Type & Weight & $\mathrm{M}_{n}$ & Weight & Weight fraction & Volume fraction & \\
\hline M129 & MMA & 70 & 1700 & 30 & 0.25 & 0.29 & Transparent \\
\hline M147 & MMA & 50 & 1700 & 50 & 0.42 & 0.47 & Transparent \\
\hline M432 & MMA & 70 & 4700 & 30 & 0.28 & 0.32 & Opaque \\
\hline M442 & MMA & 60 & 4700 & 40 & 0.38 & 0.42 & Opaque \\
\hline T 436 & TFEMA & 70 & 4700 & 30 & 0.28 & 0.36 & Transparent \\
\hline T 446 & TFEMA & 60 & 4700 & 40 & 0.38 & 0.46 & Transparent \\
\hline
\end{tabular}

Abbreviations: PDMS, polydimethylsiloxane; MMA, methylmethacrylate; TEEMA, trifluoroethylmethacrylate. aparts of weight for $1 G$ is Acrylic monomer $\times(1 / 100)$ for all code.

bPDMS weight fraction and volume fraction were calculated using PDMS weight, which was obtained from the subtraction of the weight of methacryloyloxypropyl group from PDMS macromonomer.

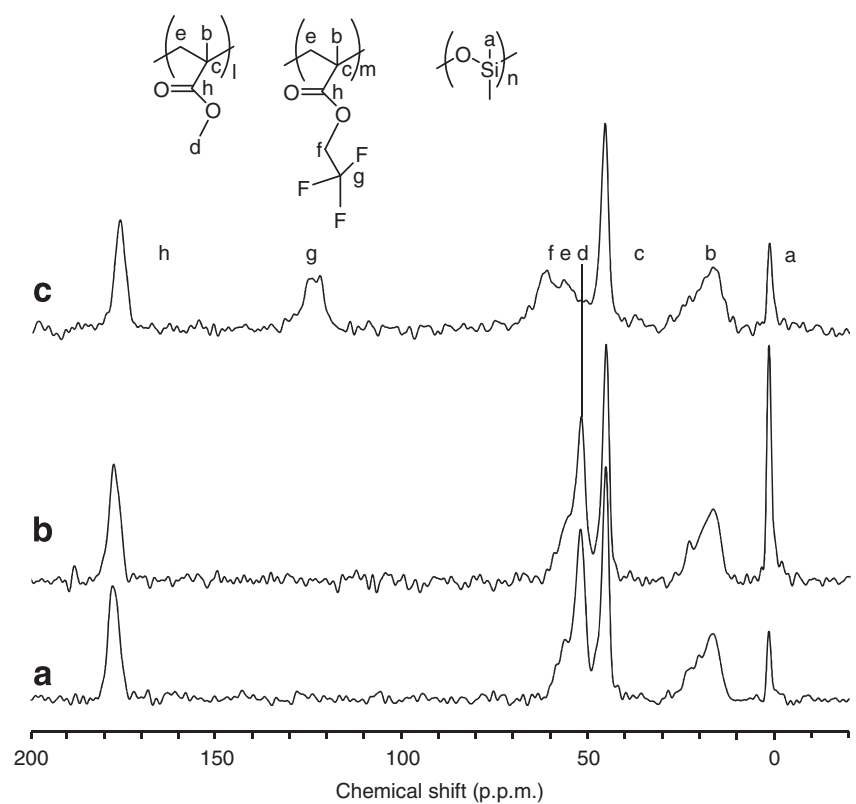

Figure $2{ }^{13} \mathrm{C}$ CP-MAS NMR spectra of crosslinked copolymers from methacrylates and the PDMS macromonomer. $\mathbf{a}, \mathbf{b}$, and $\mathbf{c}$ correspond to the following compositions. $\mathbf{a}$ : M442 (MMA/PDMS macromonomer with $M_{\mathrm{n}}=4700, \mathrm{PDMS}=38 \mathrm{wt} \%$ ), b: M147 (MMA/PDMS macromonomer with $M_{\mathrm{n}}=1700, \mathrm{PDMS}=42$ wt $\%$ ), c: T446 (TFEMA/PDMS macromonomer with $M_{\mathrm{n}}=4700, \mathrm{PDMS}=38$ wt\%). was higher than those of specimens M442 and T446, with a PDMS $M_{\mathrm{n}}$ of 4700 . It is reported that because the efficiency of crosspolarization inversely depends on mobility, a higher intensity signal corresponds to a lower mobility in the CP-MAS spectrum. ${ }^{14}$ Because the PDMS content is almost the same for all specimens (PDMS is 38-42 wt \%), it was recognized from the signal at 1.5 p.p.m. (Figure 2a) that the larger PDMS segment $\left(M_{\mathrm{n}}=4700\right)$ is more mobile than the PDMS segment with an $M_{\mathrm{n}}$ of 1700 . These results suggest that a higher PDMS $M_{\mathrm{n}}$ allows a higher diffusivity of gas molecules and results in a higher gas permeability. This shows good correspondence to our previous report that described a material with a microheterophase structure with a high $M_{\mathrm{n}}$ that showed a higher oxygen permeability than a material made from a low-molecular-weight silicone constituent. ${ }^{10}$

\section{Comparison of $T_{1}$ values from the} copolymer and pristine polymer The measured $T_{1}{ }^{\mathrm{H}}, T_{1 \rho}{ }^{\mathrm{H}}, T_{1}{ }^{\mathrm{C}}$ and $T_{1 \rho}{ }^{\mathrm{C}}$ values of pristine PDMS (I), PMMA (I) and PTFEMA (II) are shown with those of the copolymer in Figure 3a, b, c and d. The $T_{1}{ }^{\mathrm{H}}$, $T_{1 \rho}{ }^{\mathrm{H}}, T_{1}{ }^{\mathrm{C}}$ and $T_{1 \rho}{ }^{\mathrm{C}}$ values of pristine PDMS are clearly different from those of copolymerized PDMS. Furthermore, comparing the $T_{1}$ values of pristine PMMA and PTFEMA with those of copolymerized PMMA and PTFEMA, a composition dependency of the $T_{1}$ values can be seen. Thus, it was recognized that the copolymerization of the PDMS macromonomer with MMA or TFEMA resulted in a finely dispersed microstructure that is dependent on the composition and PDMS $M_{\mathrm{n}}$ value.

$T_{1}{ }^{\mathrm{H}}$ vs PDMS $M_{\mathrm{n}}$, PDMS volume fraction, and co-monomer

The proton spin-lattice relaxation time $T_{1}{ }^{\mathrm{H}}$ can be used to elucidate the parts that have a relatively high mobility, on the order of $\mathrm{MHz}$, and $T_{1} \mathrm{H}$ measurements have been performed to obtain information with respect to the size of the domains in polymers. ${ }^{15-18}$ The measured $T_{1}{ }^{\mathrm{H}}$ values for all specimens, including pristine PDMS, PMMA and PTFEMA, are shown in Figure $3 \mathrm{a}$. In addition, the $T_{1}{ }^{\mathrm{H}}$ values for the PDMS macromonomer with $M_{\mathrm{n}}=1700$ and 4700 were $1.16 \mathrm{~s}$ and $1.25 \mathrm{~s}$, respectively.

Specimen M129 (MMA copolymer and $1700 M_{\mathrm{n}}$ PDMS) had almost the same $T_{1}{ }^{\mathrm{H}}$ values at all peaks (average value is $0.60 \mathrm{~s}$ ), implying effective spin diffusion due to the low molecular mobility and implying a homogeneous dispersion structure. The diameter of the homogeneously distributed area 

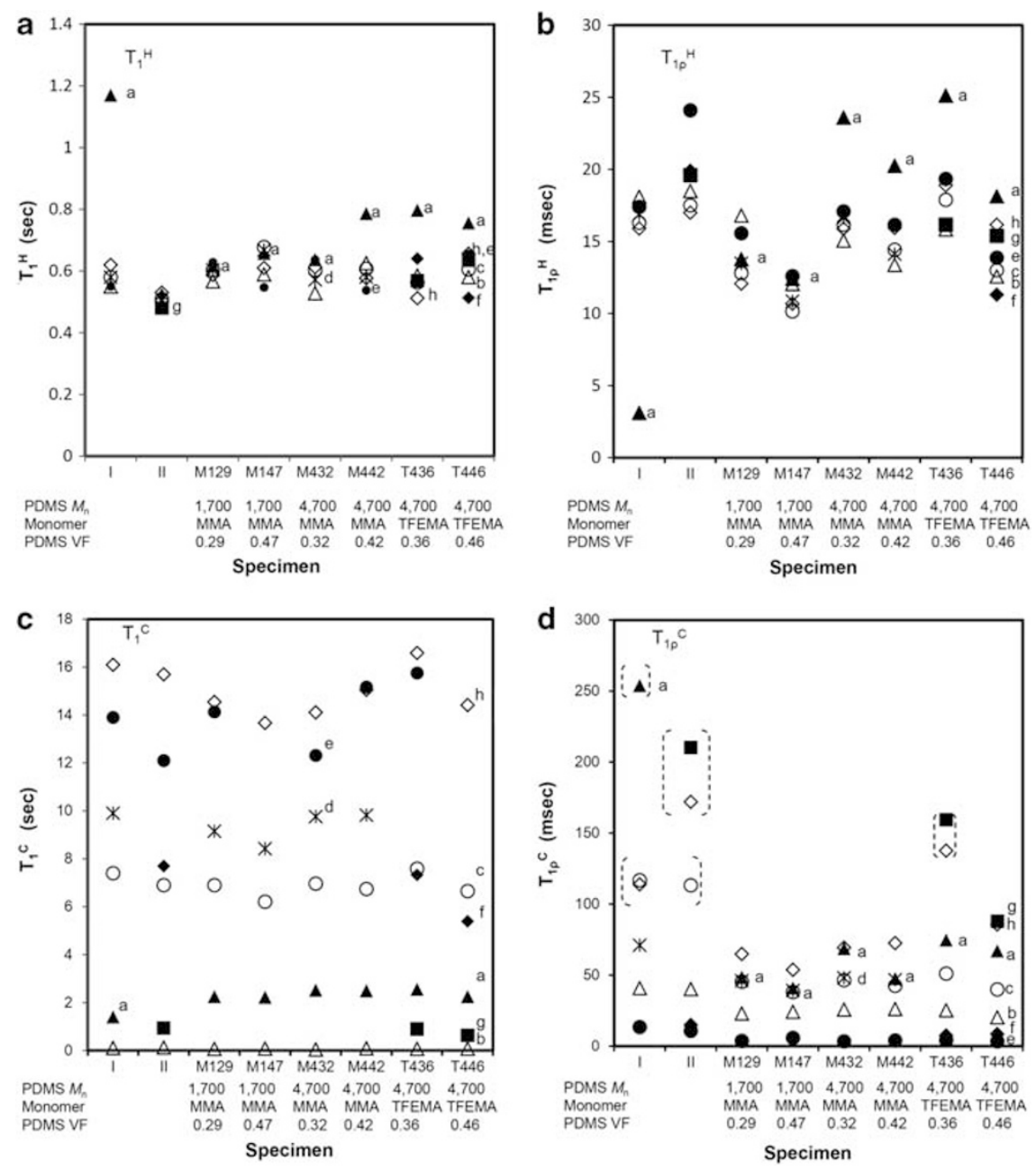

Figure 3 Measured $T_{1}{ }^{\mathrm{H}}$ (a), $T_{1 p}{ }^{\mathrm{H}}(\mathbf{b}), T_{1}{ }^{\mathrm{C}}$ (c) and $T_{1 p}{ }^{\mathrm{C}}$ (d) values. a, b, c, d, e, f, g, and h correspond to the peak signals in Figure 2. PDMS VF indicates the PDMS volume fraction. The dotted-line brackets in (d) show only the trends in the measured values and not the exact values. I and II indicate pristine PDMS $\left(M_{\mathrm{n}}=4700\right)$ combined with pristine PMMA, and pristine PTFEMA, respectively.

(domain size) was calculated using the following formula ${ }^{16}$ and average $T_{1}{ }^{\mathrm{H}}$ value:

$$
\mathrm{L}=(6 \mathrm{Dt})^{1 / 2}
$$

where $\mathrm{L}$ represents the domain size, $\mathrm{D}$ is $\sim 10^{-12} \mathrm{~cm}^{2} \mathrm{~s}^{-1}$ (spin diffusion coefficient), and t represents $T_{1} \mathrm{H}$.

Thus, this implies that in M129, PDMS and PMMA are dispersed homogeneously within regions that are $19 \mathrm{~nm}$ in diameter. Similarly, the specimens M147 and M432, which had different PDMS $M_{\mathrm{n}}$ values, showed $T_{1}{ }^{\mathrm{H}}$ values with relatively small dispersion, and both were determined to have a similar diameter of homogeneously dispersed range.

Alternatively, $T_{1}{ }^{\mathrm{H}}$ values at peak (a) for the specimens M442, T436 and T446, all with an $M_{\mathrm{n}}$ of 4700 , showed higher values than those of M129, M147 and M432. Because longer $T_{1}{ }^{\mathrm{H}}$ values correspond to higher degrees of molecular motion, this implies that a larger PDMS $M_{\mathrm{n}}$ results in more molecular motion, a higher diffusivity of gas molecules and a higher gas permeability, similar to what was observed using the CPMAS spectra. A longer $T_{1}{ }^{\mathrm{H}}$ value also corresponds to inefficient spin diffusion and results in phase separation of PDMS and PMMA or PTFEMA. Therefore, these results correspond well with our previous report. ${ }^{10}$

MMA copolymers with a higher PDMS content showed higher values of $T_{1}{ }^{\mathrm{H}}$ at peak (a), as shown in Figure 3a. Alternatively, TFEMA copolymers with a higher PDMS content, T446, showed lower $T_{1}{ }^{\mathrm{H}}$ values. At the same time, the dispersion of the $T_{1}{ }^{\mathrm{H}}$ values of all peaks of T446 was smaller than that of T436, suggesting differences in the microstructure between T446 and other copolymers. Previously, different morphologies have been reported from copolymers with different ratios of constituents or different $M_{\mathrm{n}}$ values using TEM observations of the acrylate/PDMS macromonomer copolymer. ${ }^{9}$ Therefore, the aforementioned results suggest that a similar morphology in the TFEMA copolymer may appear at a lower PDMS content than that in the MMA copolymer. Furthermore, the smaller dispersion of $T_{1}{ }^{\mathrm{H}}$ values of all peaks from T446 implies the finer structure or more continuous structure in T446 than that in T436 or M442. Thus, resulting differences in gas permeability for MMA copolymers and TFEMA copolymers can be expected. Therefore, the reported oxygen permeability increase ${ }^{11}$ can be supported by the possible differences in morphology.

These observations with respect to the correlation between morphology and dispersion of $T_{1}{ }^{\mathrm{H}}$ values could explain the $T_{1}{ }^{\mathrm{H}}$ value at peak (a) in M432. 
$T_{1 \rho}{ }^{\mathrm{H}}$ vs PDMS $M_{n}$, PDMS volume fraction, and co-monomer

The $T_{1 \rho}{ }^{\mathrm{H}}$ value reflects motion modes of the order of several $\mathrm{kHz}$, which appear primarily in the main chain of the polymer. The relationship between the spin-diffusion phenomenon and molecular mobility is the same as with $T_{1} \mathrm{H}$.

As shown in Figure 3b, specimen M147, with a PDMS $M_{\mathrm{n}}$ of 1700 and a PDMS volume fraction of 0.47 , showed minimum dispersion among all $T_{1 \rho}{ }^{\mathrm{H}}$ values. Because this implies effective spin diffusion, the diameter of the homogeneously distributed area (domain size) was calculated to be $2.6 \mathrm{~nm}$ using equation (1). This suggests that in M147, PDMS and PMMA are dispersed homogeneously in an area with a diameter of $2.6 \mathrm{~nm}$. Similarly, the dispersion structure of M129 is considered to be the same as that of specimen M147, suggesting that the 1700 $M_{\mathrm{n}}$ PDMS macromonomer did not influence the microphase separation when the PDMS volume fraction ranged from 0.29 to 0.47 . This suggests that a smaller $M_{\mathrm{n}}$ results in a transparent copolymer and could explain the results related to copolymer transparency shown in Table 1.

Comparison of the $T_{1 \rho}{ }^{\mathrm{H}}$ values of specimens with different PDMS $M_{\mathrm{n}}$ values suggested that a larger PDMS $M_{\mathrm{n}}$ value caused higher $T_{1 \rho}{ }^{\mathrm{H}}$ values at peak (a) from 13.8 and $12.4 \mathrm{~ms}$ to 23.6, 20.2, 25.1 and $18.1 \mathrm{~ms}$. Specifically, the influence of $-\mathrm{OSi}\left(\mathrm{CH}_{3}\right)_{2}$ chain length on the molecular mobility and gas permeability can be observed using the $T_{1 \rho}{ }^{\mathrm{H}}$ value and the $T_{1}{ }^{\mathrm{H}}$ value.

As shown in Figure 3b, a comparison of the $T_{1 \rho}{ }^{\mathrm{H}}$ values at peak (a) of the specimens with different PDMS volume fractions showed that higher PDMS volume fractions resulted in lower $T_{1 \rho}{ }^{\mathrm{H}}$ values. The magnitude of change in the $T_{1 \rho} \mathrm{H}$ value depended on the PDMS $M_{\mathrm{n}}$ and co-monomer type. It is interesting that the nature of the $T_{1 \rho}{ }^{\mathrm{H}}$ dependency on PDMS content is opposite to that of $T_{1}{ }^{\mathrm{H}}$.

Furthermore, T446 displayed a somewhat reduced dispersion of all $T_{1 \rho}{ }^{\mathrm{H}}$ values when compared with the distinct separation of $T_{1 \rho}{ }^{\mathrm{H}}$ values between peak (a) and the other peaks (b), (c), (e), (f), (g) and (h) in T436. In addition, it is interesting that the $T_{1 \rho}{ }^{\mathrm{H}}$ values at peak (g) ( $\mathrm{C}$ in $\mathrm{CF}_{3}$ group) and peak $(\mathrm{h})(\mathrm{C}$ in $\mathrm{C}=\mathrm{O}$ group) are close in value to those of peak (a) in T446. A comparison of $T_{1 \rho}{ }^{\mathrm{H}}$ for T446 and M442 led to the same result. These results can be correlated to the possible existence of domain dispersion of the order of several nm or another morphology with finer structures or more continuous structures in T446 than those in T436 or M442. These differences in morphology could lead to the observed difference in gas permeability and support the previously reported results. ${ }^{11}$

\section{$T_{1}^{\mathrm{C}}$ Values}

Results from the $T_{1}{ }^{\mathrm{C}}$ measurements are shown in Figure $3 \mathrm{c}$. Because the spin-diffusion phenomenon does not occur in the ${ }^{13} \mathrm{C}$ nucleus, independent $T_{1}{ }^{\mathrm{C}}$ values for each $\mathrm{C}$ atom were available. This relaxation time also reflects the molecular motion at a frequency of several MHz. Comparing the $T_{1}{ }^{\mathrm{C}}$ data from M129 and M147, both with a PDMS $M_{\mathrm{n}}$ of 1700 , it was determined that the $T_{1}{ }^{\mathrm{C}}$ values of peaks (c), (d) and (h) decreased with an increase in PDMS content. Differences between M432 and M442, both with a PDMS $M_{\mathrm{n}}$ of 4700 , could not be found. However, the decrease in $T_{1}{ }^{\mathrm{C}}$ from peaks (c), (f) and (h) for specimens T436 and T446, both with a PDMS $M_{\mathrm{n}}$ of 4700 , and the PTFEMA copolymer, was recognized with an increase in PDMS content. The decrease in $T_{1}{ }^{\mathrm{C}}$ means an increase in the molecular mobility because these peaks result from PMMA or PTFEMA that have a high $T_{\mathrm{g}}$ value and the applicability of the relationship $\omega_{\mathrm{c}} \tau \gg 1$, where $\omega_{\mathrm{c}}$ is angular velocity of the ${ }^{13} \mathrm{C}$ nucleus and $\tau$ is the correlation time. Therefore, these $T_{1}{ }^{\mathrm{C}}$ values suggest a difference in the mobility of PMMA or PTFEMA when comparing the M129 and M147, as well as T436 and T446.

\section{$T_{1 \rho}{ }^{\mathrm{C}}$ vs the PDMS $M_{\mathrm{n}}$ value, the PDMS} volume fraction, and the co-monomer $T_{1 \rho}{ }^{\mathrm{C}}$ yields information with respect to molecular motion on the order of several $\mathrm{kHz}$, similar to $T_{1 \rho}{ }^{\mathrm{H}}$, and independent $T_{1 \rho}{ }^{\mathrm{C}}$ values are available. Results from the measurements are shown in Figure 3d. $T_{1 \rho}{ }^{\mathrm{C}}$ values from peak (a) for pristine PDMS, peaks (c) and (h) for pristine PMMA and PTFEMA, peak (g) for pristine PTFEMA, and peaks $(\mathrm{g})$ and $(\mathrm{h})$ for T436 are plotted only to demonstrate the trends in the measured values and are not exact values because of possible measurement errors.

From Figure $3 \mathrm{~d}$, it was suggested that a higher $T_{1 \rho}{ }^{\mathrm{C}}$ value at peak (a) was obtained in specimens with a larger PDMS $M_{\mathrm{n}}$ when the PDMS volume fraction ranged from 0.29 to 0.36 . In other words, it is suggested that the longer - $\mathrm{OSi}\left(\mathrm{CH}_{3}\right)_{2}$ - chain resulted in higher molecular mobility and in higher gas molecule permeability, as was also observed in the $T_{1}{ }^{\mathrm{H}}$ and $T_{1 \rho}{ }^{\mathrm{H}}$ measurements. Moreover, it is interesting that the $T_{1 \rho}{ }^{\mathrm{C}}$ value at peak (a) in the TFEMA/PDMS copolymer is larger than that of the MMA/PDMS copolymer and that the $T_{1 \rho}{ }^{\mathrm{C}}$ values at peak $(\mathrm{g})$ and $(\mathrm{h})$ are larger than that at peak (a). This implies amplified molecular motion in the - $\mathrm{OSi}\left(\mathrm{CH}_{3}\right)_{2}$ - chain,
$-\mathrm{CF}_{3}$ group and $-\mathrm{C}=\mathrm{O}$ group and results in a large contribution from the fluorine-containing group to the oxygen permeability, as has been previously reported. ${ }^{11}$

Phase size and phase separation estimation Specimens containing PDMS with an $M_{\mathrm{n}}$ of 1700 exhibited a homogeneously dispersed structure with a diameter of $2.6 \mathrm{~nm}$. When PDMS with an $M_{\mathrm{n}}$ of 4700 was employed, some samples showed a homogeneously dispersed structure with a diameter of approximately $20 \mathrm{~nm}$; therefore, from the viewpoint of a scale on the order of several $\mathrm{nm}$, all specimens had a phase-separated structure.

\section{Conclusion}

We conducted ${ }^{13} \mathrm{C}$ solid-state NMR measurements to investigate the molecular-level relationship between the physical properties and the composition of copolymers produced from methacrylates, PDMS macromonomers and crosslinkers. Using the data from $T_{1}{ }^{\mathrm{H}}$ and $T_{1 \rho} \mathrm{H}$, it was suggested that a larger PDMS $M_{\mathrm{n}}$ value resulted in a higher molecular mobility of - $\mathrm{OSi}\left(\mathrm{CH}_{3}\right)_{2-}$, and the high mobility could be correlated to a higher gas permeability. In addition, the copolymer with an $M_{n}$ of PDMS of 1700 was found to have a homogeneously dispersed structure with a diameter of approximately $3 \mathrm{~nm}$. PDMS with higher values of $M_{\mathrm{n}}$, such as $4700 M_{\mathrm{n}}$ PDMS, resulted in a phase-separated structure on the order of several $\mathrm{nm}$ and eventually resulted in a homogeneously dispersed structure with a diameter of tens of nm. This result could be correlated to the appearance of the copolymer.

Differences in the morphology of MMA copolymers and TFEMA copolymers were suggested from the $T_{1}{ }^{\mathrm{H}}, T_{1 \rho}{ }^{\mathrm{H}}$ and $T_{1 \rho}{ }^{\mathrm{C}}$ measurements. Copolymerization of $\mathrm{CF}_{3}$ bearing trifluoroethyl methacrylate resulted in a larger $T_{1 \rho}{ }^{\mathrm{C}}$ value at peak (a), and the $-\mathrm{CF}_{3}(\mathrm{~g})$ and $-\mathrm{C}=\mathrm{O}(\mathrm{h})$ groups showed higher $T_{1 \rho}{ }^{\mathrm{C}}$ values than that of the $\mathrm{OSi}\left(\mathrm{CH}_{3}\right)_{2^{-}}$chain. The described results implied that the amplified molecular motion of aforementioned groups is related to the mechanism of increased oxygen permeability in copolymers produced from fluorine-bearing monomers and the PDMS macromonomer.

\section{ACKNOWLEDGEMENTS}

This work was partly supported by The Foundation of Showa Hokokai.

Mitsuru Yokota ${ }^{1}$, Yuko Miwa ${ }^{2}$, Hiroharu Ajiro $^{1,3}$ and Mitsuru Akashi ${ }^{1,3}$

${ }^{1}$ Department of Applied Chemistry, Graduate School of Engineering, Osaka 
University, Suita, Japan; ${ }^{2}$ Toray Research Center, Inc., Otsu, Japan and ${ }^{3}$ The Center for Advanced Medical Engineering and Informatics, Osaka University, Suita, Japan E-mail:akashi@chem.eng.osaka-u.ac.jp

1 Yamanaka, K. \& Inoue, T. Structure development in epoxy resin modified with Poly (ether sulphone). Polymer 30, 662-667 (1989).

2 Wang, X., Okada, M. \& Han, C. C. Polymerizationinduced phase separation of styrene and its derivatives:rarity of crystal-like array formation. Macromole cules 40, 4378-4380 (2007)

3 Kwok, A. Y., Prime, E. L., Qiao, G. G. \& Solomon, D. H. Synthetic hydrogels 2. Polymerization induced phase separation in acrylamide systems. Polymer 44, 7335-7344 (2003)

4 Matsumoto, T., Koinuma, Y., Waki, K., Kishida, A., Furuzono, T., Maruyama, I. \& Akashi, M. Novel functional polymers: poly(dimethylsiloxane)-polyamide multiblock copolymer. IV. Gas permeability and thermomechanical properties of aramid-silicone resins. J. Appl. Polym. Sci. 59, 1067-1071 (1996).
5 Ha, S. Y., Park, H. B. \& Lee, Y. M. Percolational effect of siloxane content in poly (amideimide siloxane) on the gas permeation behavior. Macromolecules 32, 2394 2396 (1999).

6 Bruns, N., Scherble, J., Hartmann, L., Thomann, R., Iván, B., Muellhaupt, R. \& Tiller, J. C. Nanophase separated amphiphilic conetwork coatings and membranes. Macromolecules 38, 2431-2438 (2005).

7 Yamamoto, K., Ito, E., Fukaya, S. \& Takagi, H. Phaseseparated conetwork structure induced by radical copolymerization of poly(dimethylsiloxane)- $\alpha, \omega$-diacrylate and $\mathrm{N}, \mathrm{N}$ - dimethyl acrylamide. Macromolecules 42, 9561-9567 (2009).

8 Yu, X., Nagarajan, M. R., Li, C., Speckhard, T. A. \& Cooper, S. L. Properties of ultraviolet cured polydimethylsiloxane urea acrylates. J. Appl. Polym. Sci. 30, 2115-2135 (1985).

9 Mazurek, M., Kinning, D. J. \& Kinoshita, T. Novel materials based on silicone-acrylate copolymer networks. J. Appl. Polym. Sci. 80, 159-180 (2001).

10 Yokota, M., Goshima, T. \& Itoh, S. The effect of polymer structure on durability of high $D k$ rigid gaspermeable materials. J. Brit. CL. Assoc. 15, 125-129 (1992).

11 Koßmehl, G., Fluthwedel, A. \& Schäfer, H. Highly oxygen-permeable copoly(methacrylate/siloxane)s with perfluoroalkyl ester groups. Makromol. Chem. 193, 157-166 (1992).
12 Havens, J. R. \& VanderHart, D. L. Morphology of poly (ethylene terephthalate) fibers as studied by multiplepulse ${ }^{1} \mathrm{H}$ NMR. Macromolecules 18, 1663-1676 (1985).

13 Clauss, J., Schmidt-Rohr, K. \& Spiess, H. W. Determination of domain size in heterogeneous polymers by solid-state NMR. Acta. Polymer. 44, 1-17 (1993).

14 Cho, G., Natansohn, A., Ho, T. \& Wynne, K. J. Phase structure of poly (dimethylsiloxane-urea-urethane)-segmented copolymers as observed by solid-state nuclear magnetic resonance spectra. Macromolecules 29, 2563-2569 (1996).

15 Asano, A., Takegoshi, K. \& Hikichi, K. ${ }^{13}$ C c.p./ m.a.s.n.m.r. study on the miscibility and phase separation of a polystyrene/poly(vinyl methyl ether) blend. Polymer 35, 5630-5636 (1994).

16 McBrierty, V. J. \& Douglass, D. C. Recent advances in the nmr of solid polymers. J. Polym. Sci. Macromol. Rev. 16, 295-366 (1981).

17 Demco, D. E., Utitu, L., Tilmann, W., Blümich, B. \& Popescu, C. Morphology and molecular dynamics of hard $\alpha$-keratin under pressure by ${ }^{1} \mathrm{H}$ and ${ }^{13} \mathrm{C}$ solid-state NMR. Chem. Phys. Lett. 509, 62-66 (2011).

18 Asano, A., Eguchi, M., Shimizu, M. \& Kurotsu, T. Miscibility and molecular motion of PMAA/PVAc blends investigated by high-resolution solid-state CPMAS ${ }^{13} \mathrm{C}$ NMR. Macromolecules 35, 8819-8824 (2002).

Supplementary Information accompanies the paper on Polymer Journal website (http://www.nature.com/pj) 